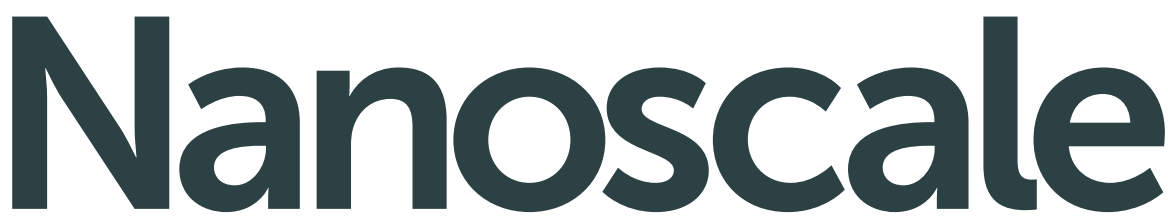

Advances

rsc.li/nanoscale-advances

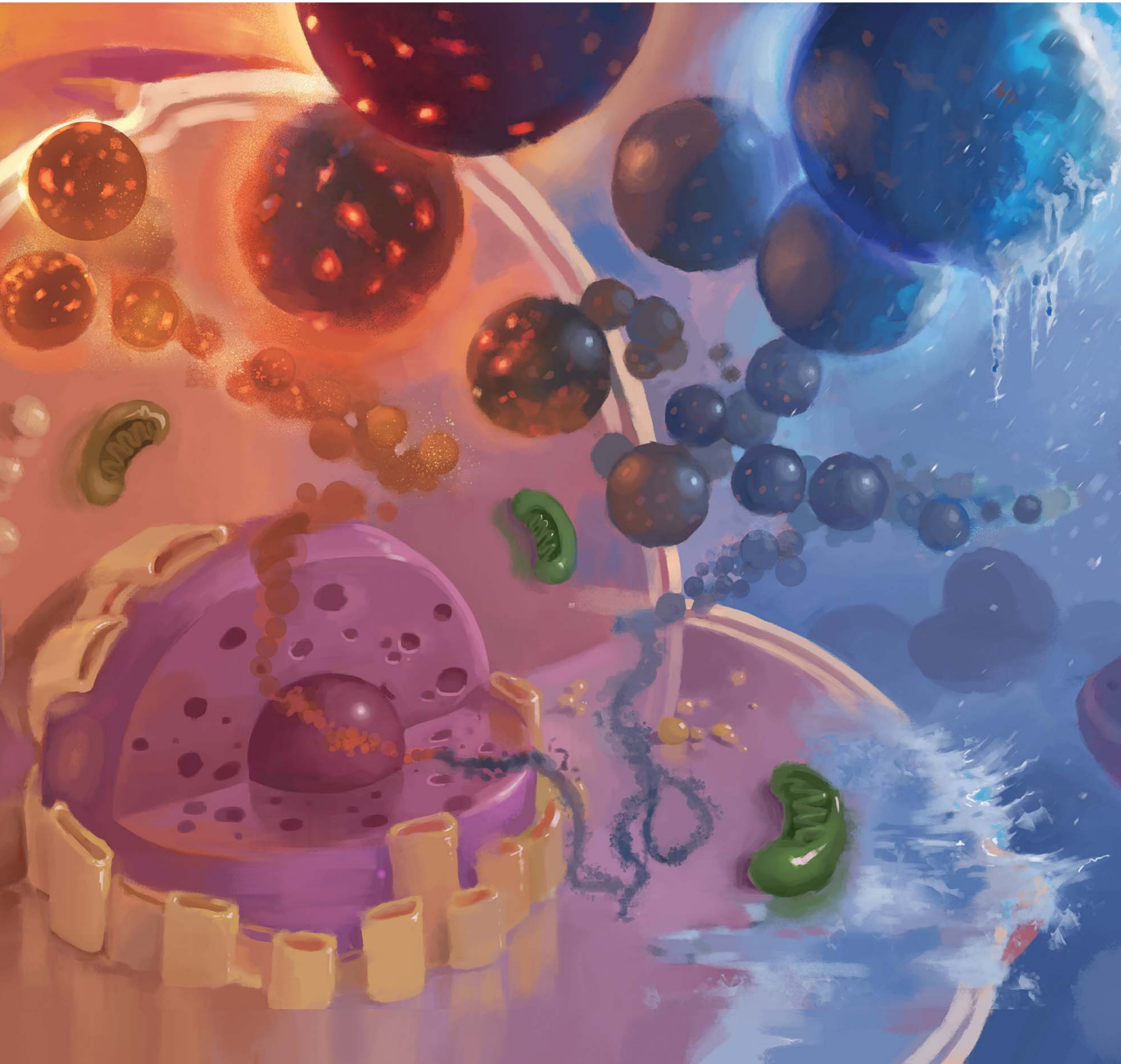

ISSN 2516-0230

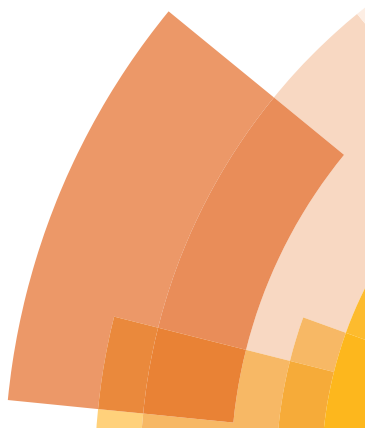


Check for updates

Cite this: Nanoscale Adv., 2019, 1, 105

\title{
Intracellular ratiometric temperature sensing using fluorescent carbon dots $\uparrow$
}

\author{
Jun-Ray Macairan, (D) a Dilan B. Jaunky, ${ }^{b}$ Alisa Piekny ${ }^{\mathrm{b}}$ and Rafik Naccache (D) *a
}

Highly sensitive non-invasive temperature sensing is critical for studying fundamental biological processes and applications in medical diagnostics. Nanoscale-based thermometers are promising non-invasive probes for precise temperature sensing with subcellular resolution. However, many of these systems have limitations as they rely on fluorescence intensity changes, deconvolution of peaks, or the use of hybrid systems to measure thermal events. To address this, we developed a fluorescence-based ratiometric temperature sensing approach using carbon dots prepared via microwave synthesis. These dots possess dual fluorescence signatures in the blue and red regions of the spectrum. We observed a linear response as a function of temperature in the range of $5-60{ }^{\circ} \mathrm{C}$ with a thermal resolution of $0.048 \mathrm{~K}^{-1}$ and thermal sensitivity of $1.97 \% \mathrm{C}^{-1}$. Temperature-dependent fluorescence was also observed in HeLa cancer cells over a range of $32-42{ }^{\circ} \mathrm{C}$ by monitoring changes in the red-to-blue fluorescence signatures. We demonstrate that the ratiometric approach is superior to intensity-based thermal sensing because it is independent of the intracellular concentration of the optical probe. These findings suggest that dual-emitting carbon dots can be an effective tool for in vitro and possibly in vivo fluorescence nanothermometry.

Received 28th September 2018 Accepted 9th December 2018

DOI: $10.1039 / \mathrm{c} 8 \mathrm{na00255j}$

rsc.li/nanoscale-advances
Moreover, they could be used in synergy with other nanomaterials such as those studied for the development of novel hyperthermia treatments. ${ }^{3,5}$ In this case, combining thermal sensing with localized heating of the diseased area would minimize damage associated with overheating of the surrounding healthy tissue..$^{20-22}$

Thermal sensing using nanomaterials can be accomplished by exploiting their optical properties. Fluorescence nanothermometry can be categorized into several classes depending on the specific parameter from which the thermal measurement is extracted including signal intensity, band-shape, fluorescence lifetimes, band-shift, excitation wavelength polarization and spectral shift, among others. ${ }^{4}$ To date, the most common fluorescent temperature-sensing probes extract temperature information by changes in signal intensity, variation in the fluorescence lifetimes, as well as band-shape. , $^{3,5,9,14,15,23,24}$ In the first case, fluorescence varies with changing temperature and may be observed as an absolute increase (or decrease) in signal. Lifetime nanothermometry is independent of signal intensity and allows for the extraction of thermal information through variation in the lifetime of the fluorescence event. Lastly, bandshape nanothermometry exploits the presence of two or more fluorescence bands. The fluorescence intensity (or area) is monitored for both bands over the temperature range and a comparative relationship is established. This method is advantageous over other methods since it is not concentrationdependent, does not rely on temperature-dependent decay curves and does not suffer from environmental interference University, Montreal, QC, Canada, H4B 1R6

$\dagger$ Electronic supplementary information (ESI) available. See DOI: 10.1039/c8na00255j

${ }^{a}$ Department of Chemistry and Biochemistry, Center for NanoScience Research, Concordia University, Montreal, QC, Canada, H4B 1R6. E-mail: rafik.naccache@

concordia.ca 
(e.g. surrounding media or environment, variability in cell uptake at different temperatures, etc.). ${ }^{18}$

Among luminescent nanomaterials, carbon dots have garnered significant attention in recent years due to their versatile optical properties, notably tunable fluorescence and high photostability. ${ }^{25-29}$ In addition, they are dispersible in water, have excellent biocompatibility and low cytotoxicity. ${ }^{25,29-34}$ As such, they can be integrated into sensing, bioimaging and diagnostic applications such as fluorescent nanothermometry. Recently, carbon dot optical probes have been investigated for temperature sensing applications in vivo and in vitro. ${ }^{9,16,23,24,35-37}$ Yang et al. ${ }^{24}$ reported the synthesis of low cytotoxic biocompatible carbon dots with temperaturedependent fluorescence intensity from 20 to $80{ }^{\circ} \mathrm{C}$ and a thermal sensitivity of $0.85 \%{ }^{\circ} \mathrm{C}^{-1}$. They extended their work to show temperature-dependent fluorescence in an in vivo mouse model. In another study, ${ }^{16}$ temperature-sensitive fluorescence lifetime carbon dot nanothermometers showed a sensitivity of $1.79 \%$ and promising results for temperature sensing in cells. In a different approach, ${ }^{23}$ a ratiometric carbon dot temperature sensing probe was developed over the range of 5 to $85{ }^{\circ} \mathrm{C}$. This was achieved via deconvolution of overlapping fluorescence bands resulting in a thermal sensitivity of $1.48 \%{ }^{\circ} \mathrm{C}^{-1}$. Wang et $a .^{38}$ circumvented the need for deconvolution of peaks through the development of nanohybrids consisting of carbon dots and gold nanoclusters, which yield blue and red emission, respectively, with a thermal sensitivity of $1.8 \%{ }^{\circ} \mathrm{C}^{-1}$.

Distinct fluorescent bands emanating from a single probe is ideal for ratiometric temperature sensing to minimize variability and ensure homogeneous physico-optical properties. Few reports have focused on dual-fluorescing carbon dots (dCDs) ${ }^{23,39-41}$ and the majority do not describe the use of these dots for fluorescence thermal sensing. To this end, we report a facile synthesis of biocompatible dCDs for fluorescence nanothermometry in live cells. Their capacity to simultaneously fluoresce in the blue and red regions of the spectrum originates from two different emissive states on the dCDs. ${ }^{23,42}$ Due to their unique optical properties, temperature sensing over the range of $5-60{ }^{\circ} \mathrm{C}$ can be accomplished via changes in fluorescence intensity or a ratiometric approach that utilizes both fluorescence signatures. The temperature sensitivity of the dCDs was assessed in colloidal dispersions and HeLa cancer cells, extending the thermometric relationship to biological systems.

\section{Results and discussion}

\section{Physico-chemical and optical characterization}

The dCDs were synthesized in a one-step microwave assistedreaction using formamide and glutathione. Quasi-spherical dots were obtained following synthesis at $180{ }^{\circ} \mathrm{C}$ for a period of 5 minutes. Transmission electron microscopy (TEM) particle characterization (Fig. 1A) show that the dots are monodisperse with an average size of $7.3 \pm 1.2 \mathrm{~nm}$. Particle size statistics obtained for a large population of the dots reveals a Gaussian size distribution (Fig. 1A inset) ranging from $\sim 5-9 \mathrm{~nm}$. Atomic force microscopy (AFM) analysis confirms our TEM findings showing that the dCDs are indeed spherical. The dCDs remain well dispersed in water and do not display any significant agglomeration upon drying on the AFM mica substrate with an average height of $1.4 \mathrm{~nm}$ (Fig. 1B inset).

We characterized the optical properties of the purified dCDs, which form a colourless dispersion in water at a concentration of $50 \mu \mathrm{g} \mathrm{mL}{ }^{-1}$. Following exposure to UV $\left(\lambda_{\text {ex }}=365 \mathrm{~nm}\right)$, a violet colour is observed due to the simultaneous contributions of blue and red fluorescence (Fig. 1C inset). UV-Vis absorbance spectroscopy reveals that the dCDs have three distinct absorption bands at 295-350 nm, 370-450 nm and 590-690 nm (Fig. 1C). The first band is assigned to the transition of $\mathrm{C}=\mathrm{C}$ bonds while the second and third bands can be ascribed to the $\pi \rightarrow \pi^{*}$ and $\mathrm{n} \rightarrow \pi^{*}$ transitions of the aromatic $\mathrm{sp}^{2}$ domains for the $\mathrm{C}=\mathrm{O}$, as well as the $\mathrm{C}=\mathrm{N} / \mathrm{C}=\mathrm{S}$ bonds, respectively. ${ }^{\mathbf{4 2 - 4 4}}$ Thus, the dCDs can be excited at multiple wavelengths in the
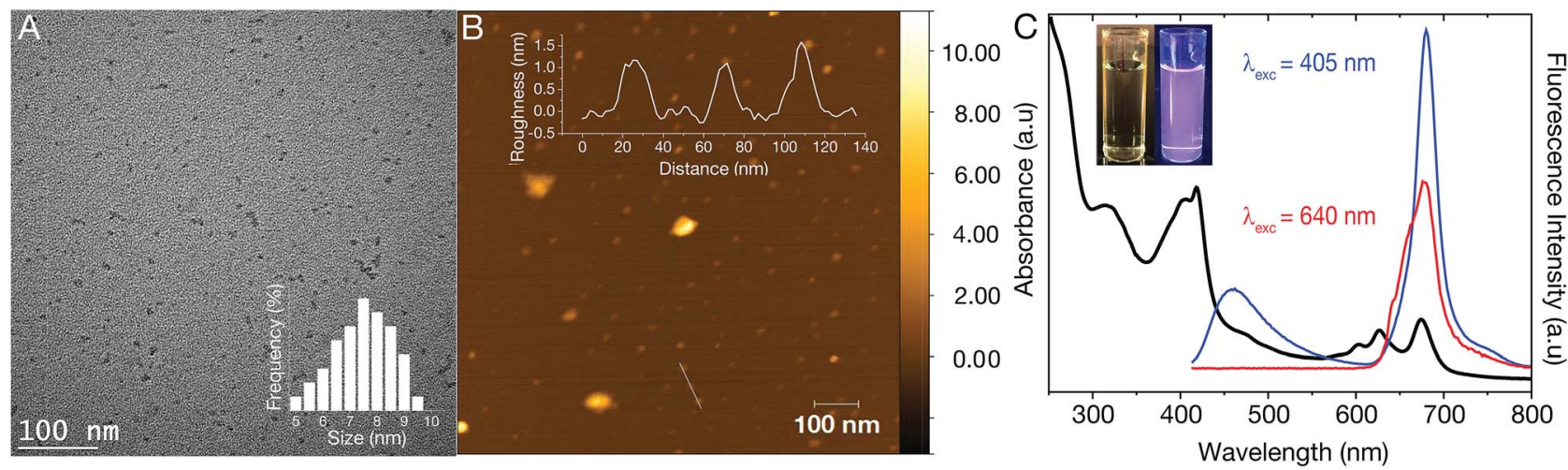

Fig. 1 (A) TEM image of a $1 \mathrm{mg} \mathrm{mL}^{-1} \mathrm{dCD}$ dispersion in water. The TEM image reveals quasi-spherical dots with a calculated particle size of $7.3 \pm$ $1.2 \mathrm{~nm}$ - inset: particle size distribution shows that the particle size spans 5-9 nm; (B) AFM image of dCDs on a mica substrate. The height profile is $1.4 \mathrm{~nm}$ as shown in the inset; (C) room temperature absorbance and fluorescence spectra of a $50 \mu \mathrm{g} \mathrm{mL} \mathrm{L}^{-1} \mathrm{dCD}$ dispersion. The UV-Vis absorption spectrum of dCDs (black curve) reveals three absorption bands centered at 295-350 nm, 370-450 nm and 590-690 nm. Following excitation at $405 \mathrm{~nm}$, two fluorescence bands are observed at 370-500 nm and 640-730 nm (blue curve) while excitation at $640 \mathrm{~nm}$ reveals red fluorescence from $645-730 \mathrm{~nm}$ (red curve); (C, inset) dCD dispersion under white light (left) and UV light ( $\lambda_{\text {ex }}=365 \mathrm{~nm}$; right). The violet color is from the contribution of both blue and red fluorescence. 
blue and red regions of the spectrum. Following excitation at $405 \mathrm{~nm}$, the dCDs exhibit two distinct fluorescence bands between 370-500 nm (blue component) and 645-730 nm (red component) as shown in Fig. 1C. Conversely, excitation at $640 \mathrm{~nm}$ only results in fluorescence in the region of 645$730 \mathrm{~nm}$. The ability to selectively excite the dCDs at longer wavelengths is attractive for bioimaging applications due to greater tissue penetration and decreased scattering. ${ }^{\mathbf{1 0 , 3 0 , 4 2 , 4 5 - 4 9}}$ Quantum yield measurements were carried out to evaluate the fluorescence efficiency of the dCDs. Following excitation at $405 \mathrm{~nm}$, a cumulative quantum yield of $6.49 \%$ was obtained using an integrating sphere with individual values of $0.38 \%$ and $6.11 \%$ ascribed to the blue and red components, respectively. This is expected since the red fluorescence dominates its blue counterpart as shown in Fig. 1C. Colloidal dCDs remain optically stable over a span of 6 months (Fig. S1†) and their optical properties do not suffer any significant degradation. The unique dual-fluorescence properties have been attributed to a mechanism consisting of carbon core and surface molecular fluorescent states. ${ }^{28}$ Herein, the blue fluorescence stems from the core of the dCDs, while the red counterpart originates from the fluorophores covalently attached to the carbon core. ${ }^{28,49}$ As shown in Fig. S2, $\uparrow$ the fluorescence stemming from the core states is excitation-dependent in the blue region of the spectrum while the excitation-independent red fluorescence originates from the molecular states. A control experiment (see Fig. S3†) was performed by separately reacting formamide and glutathione in the microwave at $180{ }^{\circ} \mathrm{C}$ for 5 min to study the optical properties of the individual precursors. No dual fluorescence was observed in this case with very weak fluorescence in the blue region of the spectrum. This highlights the necessity of both precursors for the formation of dCDs.

To assess the physico-chemical makeup of the dCDs and to understand the origins of their unique fluorescence properties, X-ray photoelectron spectroscopy (XPS) and Fourier Transform Infrared (FT-IR) analyses were carried out. The XPS survey spectrum of the dCDs (Fig. 2A) shows four binding energies at 532.08, 400.08, 286.08 and $165.08 \mathrm{eV}$ corresponding to carbon (C1s), nitrogen (N1s), oxygen (O1s) and sulfur (S2s), respectively. This observation is in accordance with what is expected given that these four elements make up the chemical composition of glutathione or formamide. High-resolution XPS (HR-XPS) analysis of the C1s binding energies and subsequent deconvolution of the observed peak reveals three components at $288.68 \mathrm{eV}, 286.89 \mathrm{eV}$ and $285.58 \mathrm{eV}$ ascribed to the presence of the $\mathrm{C}-\mathrm{C} / \mathrm{C}=\mathrm{C}, \mathrm{C}-\mathrm{O}$ and the $\mathrm{C}=\mathrm{O} / \mathrm{C}=\mathrm{N}$ functional groups, respectively (Fig. 2B). Similarly, the HR-XPS N1s spectrum (Fig. 2C) can be deconvoluted resulting in binding energies at 402.71 and $400.58 \mathrm{eV}$, which represents $\mathrm{NH}_{2} /$ pyrrolic $\mathrm{N}$, as well as graphitic $\mathrm{N}$, respectively. For O1s (Fig. 2D), the HR-XPS spectrum shows the presence of $\mathrm{C}-\mathrm{OH} / \mathrm{C}-\mathrm{OC}$ and $\mathrm{C}=\mathrm{O}$ functional groups with deconvoluted binding energies observed at 533.26 and $532.02 \mathrm{eV}$, respectively. Lastly, for S2p (Fig. 2E), three prominent binding energies are noted at 165.51, 164.14 and $163.38 \mathrm{eV}$; the former is attributed to the thiol functional groups on the dCDs while the latter is assigned to the presence of
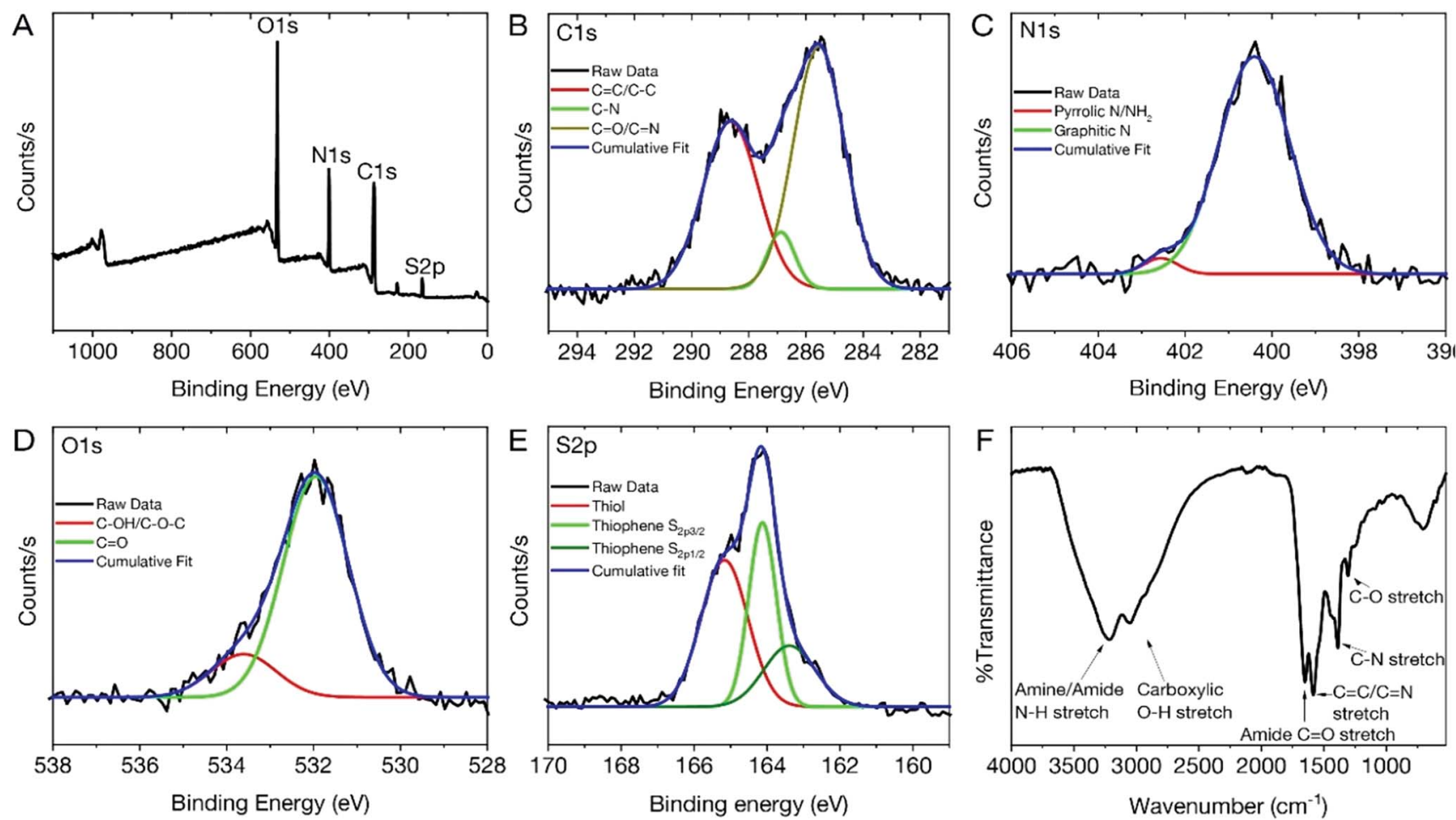

Fig. 2 (A) XPS survey spectrum of the dCDs reveals 4 binding energies ascribed to C1s, N1s, O1s and S2p. Deconvoluted HR-XPS spectra of the binding energies are ascribed to (B) C1s at a maxima of $286.08 \mathrm{eV}$; (C) N1s at a maxima of $400.08 \mathrm{eV}$; (D) O1s at a maxima of $532.08 \mathrm{eV}$; (E) S2p at a maxima of $165.08 \mathrm{eV}$; (F) FT-IR spectrum of the dCDs revealing the presence of $\mathrm{N}-\mathrm{H}$ and $\mathrm{O}-\mathrm{H}$ surface groups along with amide and carbonyl stretches. 
thiophene groups. The data confirm the presence of $\mathrm{C}=\mathrm{O}$, $\mathrm{C}=\mathrm{N}$ and $\mathrm{C}=\mathrm{S}$, which are believed to be found in the conjugated network responsible for the red absorption/emission shown in Fig. 1C. ${ }^{\mathbf{4 2 4 3}}$ The dCDs are comprised of $53.1 \%$ carbon, $26.1 \%$ oxygen, $17.4 \%$ nitrogen and $3.4 \%$ sulfur as determined using HR-XPS spectra. Our XPS assignments are corroborated by FT-IR analysis (Fig. 2F). The presence of a broad peak from $3000-3500 \mathrm{~cm}^{-1}$ is assigned to the $\mathrm{N}-\mathrm{H}$ and $\mathrm{O}-\mathrm{H}$ stretching vibrations originating from hydroxyl and amine groups, while stretches at 1645 and $1583 \mathrm{~cm}^{-1}$ reveal the presence of $\mathrm{C}=\mathrm{O}$ of an amide and of $\mathrm{C}=\mathrm{C} / \mathrm{C}=\mathrm{N}$ bonds, respectively. The presence of an amide bond is further confirmed via observation of stretches at $1386 \mathrm{~cm}^{-1}$ and $1307 \mathrm{~cm}^{-1}$, which are attributed to the $\mathrm{C}-\mathrm{N}$ amide bonds. The thiol stretch is not observed in the FT-IR spectrum, which is expected since this stretching vibration is characteristically weak in intensity.

\section{Temperature-dependent fluorescence}

We found that the fluorescent properties of aqueous colloidal dispersions of dCDs are temperature-dependent. Following excitation at $640 \mathrm{~nm}$, the fluorescence intensity and integrated area increase over the range of $5-60{ }^{\circ} \mathrm{C}$ by a factor of 3.5 (Fig. 3A). As shown in Fig. 3B, a linear response $\left(R^{2}=0.999\right)$ is observed over the entire analysis range and the temperature sensitivity was determined to be as high as $3.71 \%{ }^{\circ} \mathrm{C}^{-1}$. This value is comparable with various other polymer- and quantum dot-based nanothermometers. ${ }^{\mathbf{5 0}}$

The temperature-dependent fluorescence was also studied following excitation at $405 \mathrm{~nm}$. Interestingly, the blue and red
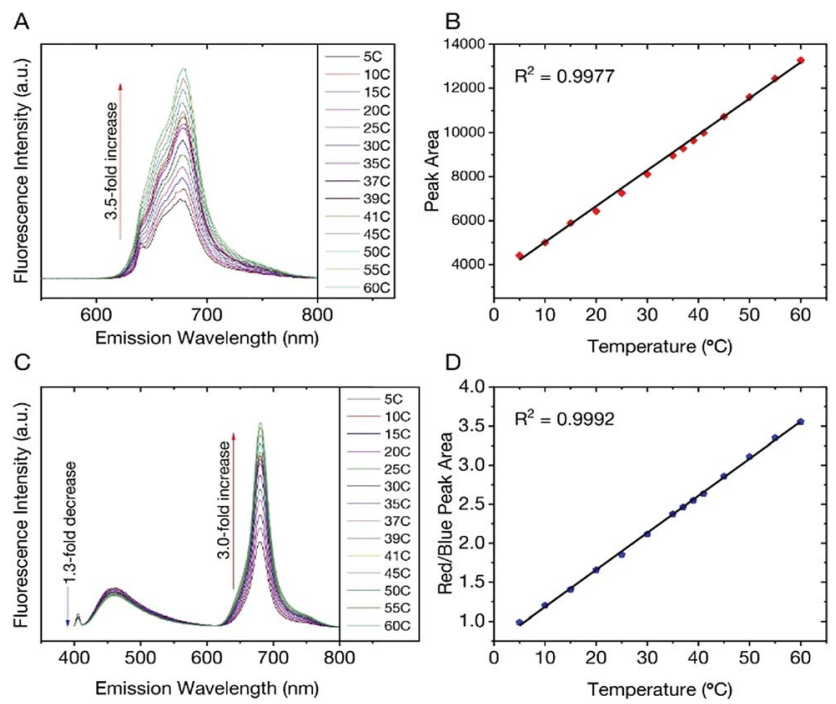

Fig. 3 (A) Excitation at $640 \mathrm{~nm}$ yields a 3.5-fold increase in fluorescence intensity and the corresponding integrated area is plotted in (B) showing a linear response over the range of $5-60{ }^{\circ} \mathrm{C}$; $(\mathrm{C})$ changes in the fluorescence spectra of the dCDs $\left(\lambda_{\text {ex }}=405 \mathrm{~nm}\right)$ as a function of temperature over the entire range. A 1.3-fold decrease is noted for the blue fluorescence in contrast to the 3-fold increase for the red counterpart; (D) the ratio of the integrated areas of the red and blue fluorescence components are plotted as a function of temperature showing a linear increase over the entire temperature range. fluorescence bands are not equally sensitive to the change in temperature. With increasing temperature, the fluorescence intensity (and corresponding integrated area under the curve) of the blue component shows a very slight decrease in contrast to the red component, which significantly increases (Fig. 3C). These observations are noted over the range of $5-60{ }^{\circ} \mathrm{C}$ where the blue emission decreases by a factor of 1.3 in contrast to the red emission, which increases by a factor of 3.0. Using eqn (1), a red-to-blue fluorescence ratio is obtained for every analysis temperature.

$$
R=\frac{\text { Integrated area of the red fluorescence }\left(\lambda_{\mathrm{ex}}=405 \mathrm{~nm}\right)}{\text { Integrated area of the blue fluorescence }\left(\lambda_{\mathrm{ex}}=405 \mathrm{~nm}\right)}
$$

As shown in Fig. 3D, the ratio of red to blue fluorescence increases with temperature and a highly linear response is observed with an $R^{2}=0.998$. These analyses were repeated in triplicate on 3 unique samples and the linear plot reflects the average of these measurements, which have small deviations at each temperature. The thermal sensitivity of the dCDs, over the entire temperature range, varied from $1.33-4.81 \%{ }^{\circ} \mathrm{C}^{-1}$, which is an improvement over previously reported carbon dot nanothermometry systems ${ }^{\mathbf{1 6 , 2 3}}$ and other dual-emitting nanomaterials such as quantum dots and metal organic frameworksdye composites. ${ }^{51-53}$ The thermal resolution of the dCDs was calculated to be $0.048 \mathrm{~K}^{-1}$ indicating that it is indeed possible to measure small thermal changes. Our system is unique in comparison to other carbon dot systems since the dual fluorescence originates from the same probe and does not require the addition of a second fluorescent probe. It should be noted that the fluorescence response of the dCDs, following a thermal event, is quick thus making it an ideal candidate for real-time temperature monitoring. As shown in Fig. S4, $\uparrow$ the fluorescence ratio stabilizes after only $2 \mathrm{~min}$, which is the time required for the entire volume of the cuvette to reach thermal equilibrium.

The reversibility and stability of the temperature-dependent fluorescence of dCDs were evaluated through thermal cycling experiments (Fig. S5 $\dagger$ ). The thermal reversibility of dCDs was assessed by measuring recovery through multiple heating and cooling cycles from 5 to $60{ }^{\circ} \mathrm{C}$. The results show that the initial fluorescence intensity does not significantly change following multiple heating and cooling cycles indicating their ability to recover following a thermal event.

\section{Cell uptake and cytotoxicity of dCDs}

We extended our study of the temperature-dependent optical properties of dCDs in cultured mammalian cells to validate their use in a model biological system. Prior to evaluating their temperature-sensing capabilities, the cytotoxicity of dCDs was assessed in HeLa cervical cancer cells. As shown in Fig. 4A, viability assays were performed using increasing concentrations of dCDs (from $0-1000 \mu \mathrm{g} \mathrm{mL} \mathrm{m}^{-1}$ ) for 48 hours. Cells remained viable up to a concentration of $100 \mu \mathrm{g} \mathrm{mL}^{-1}$ of the dCDs with an $\mathrm{IC}_{50}$ value (i.e. concentration at which $50 \%$ of the population 

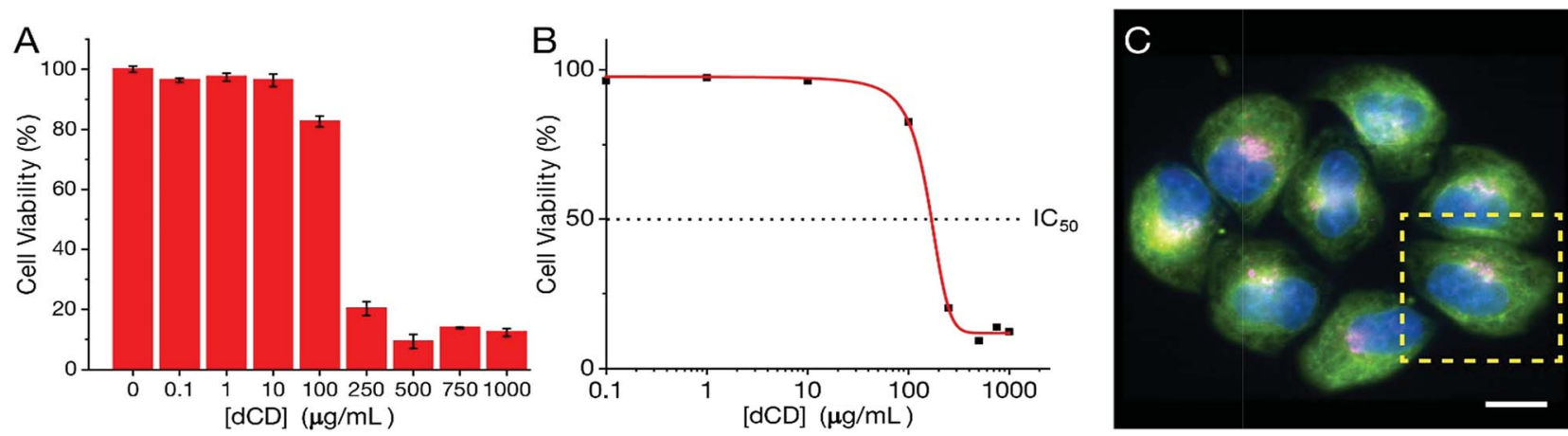

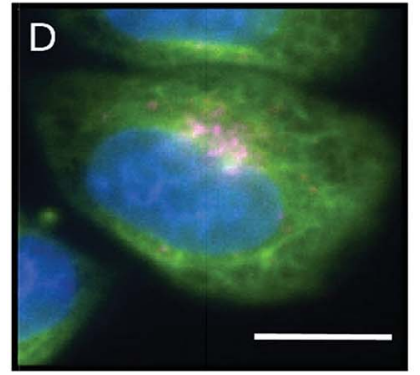

Carbon Dots/Nucleus/ Microtubules

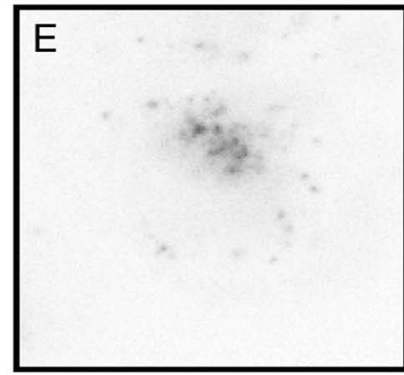

Carbon Dots

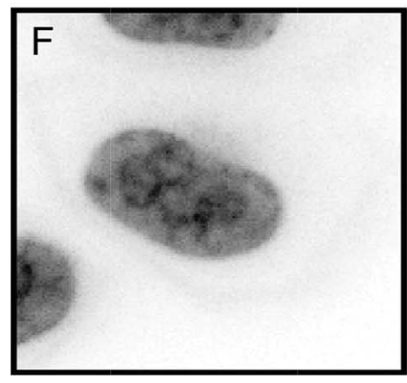

Nucleus

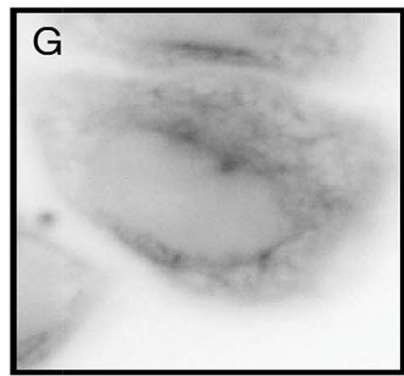

Microtubules

Fig. 4 The viability and localization of dCDs in HeLa cells $48 \mathrm{~h}$ after treatment is shown. (A) The bar graph shows the proportion of live HeLa cells treated with different concentrations of $\mathrm{dCD}$ s as indicated on the $x$-axis; (B) an IC 50 graph using log-scale shows HeLa cancer cell viability, which was measured to be $147.8 \mu \mathrm{g} \mathrm{mL}^{-1}$ (dotted line); (C) fluorescence microscopy image showing dCD-treated HeLa cancer cells co-stained with Hoechst to label DNA (blue) and Tubulin Tracker ${ }^{\mathrm{TM}}$ Green to label microtubules (green). The scale bar corresponds to a length of $10 \mu \mathrm{m}$; (D) a magnified image of a single cell (dashed box in C) shows the nucleus (DNA; blue), microtubules (green) and red-emitting dCDs. Inverted greyscale images show the (E) carbon dots, (F) nucleus and (G) microtubules.

remains) of $147.8 \mu \mathrm{g} \mathrm{mL}^{-1}$ (Fig. 4B). This suggests that the dCDs have low cytotoxicity and are biocompatible.

To determine their fluorescent properties and subcellular location, HeLa cells were treated with $100 \mu \mathrm{g} \mathrm{mL} \mathrm{m}^{-1}$ of dCDs for 24 hours to allow for cellular uptake and then imaged using fluorescence microscopy at $640 \mathrm{~nm}$ (Fig. 4C-G). The cells survived and maintained their integrity over 24 hours in support of the dCDs' low cytotoxicity (see Fig. S6†). Interestingly, the dCDs accumulated in regions that likely correspond to the endomembrane network. This network is typically found surrounding and adjacent to the nucleus (shown in blue; Fig. 4D-F). Considering that the localization of the dCDs is specific to this perinuclear region (endomembrane network), the fluorescence remains unaffected by any variation in $\mathrm{pH}$. In HeLa cells, the pH of the endomembrane network is approximately 6.6, while that of the cytoplasm is typically at 7.0-7.4. ${ }^{54}$ We have studied the dependence of fluorescence on the $\mathrm{pH}$ of the medium (Fig. $\mathrm{S} 7 \dagger$ ) and it was observed that at $\mathrm{pH}$ values between 4 and 8, there are no significant changes to the fluorescence ratios for the dCDs. As such, the reported ratios measured in both the cuvette and intracellular models are reliable.

\section{Intracellular temperature-dependent fluorescence}

To determine if the dCDs could sense temperature changes inside the cells, in vitro thermal sensing using both intensity and ratiometric approaches were performed. HeLa cells treated with dCDs were allowed to equilibrate at 32,37 and $42{ }^{\circ} \mathrm{C}$ (see Fig. 5, and $\mathrm{S} 8 \dagger$ for controls at each temperature). Excitation at $640 \mathrm{~nm}$ allows us to selectively monitor the red fluorescence of the dCDs in cells. As shown in Fig. S9, $\dagger$ the thermal changes did not correlate with a change in intensity $\left(\lambda_{\mathrm{ex}}=640 \mathrm{~nm}\right)$. This could be due to changes in intracellular concentration or localization of the dCDs at higher temperature. Thus, simply relying on changes in fluorescence intensity does lead to accurate intracellular thermal sensing.

In contrast, we do not have these limitations using the ratiometric approach. The dCDs maintain dual blue and red fluorescence in cells following excitation at $405 \mathrm{~nm}$, as previously observed for the colloidal dispersions (Fig. 5). The red-toblue ratio increases with increasing temperature, with values of 1.8 at $32{ }^{\circ} \mathrm{C}, 2.0$ at $37{ }^{\circ} \mathrm{C}$ and 2.3 at $42{ }^{\circ} \mathrm{C}$. Strikingly, these values are comparable to those observed in the cuvette model suggesting that the ratiometric relationship of the red-to-blue fluorescence of the dCDs is maintained in cells, as shown in Fig. S10. $\dagger$ This highlights the advantage of ratiometric temperature sensing in the development of fluorescent nanothermometry probes. Regardless of the amount of dCDs taken up by the cells, which can be affected by various factors such as confluency, the relative red-to-blue emission ratio remains unaffected and is not concentration dependent. Lastly, the dCDs have shown fluorescence reversibility with respect to changes in intracellular temperature. Following incubation in 


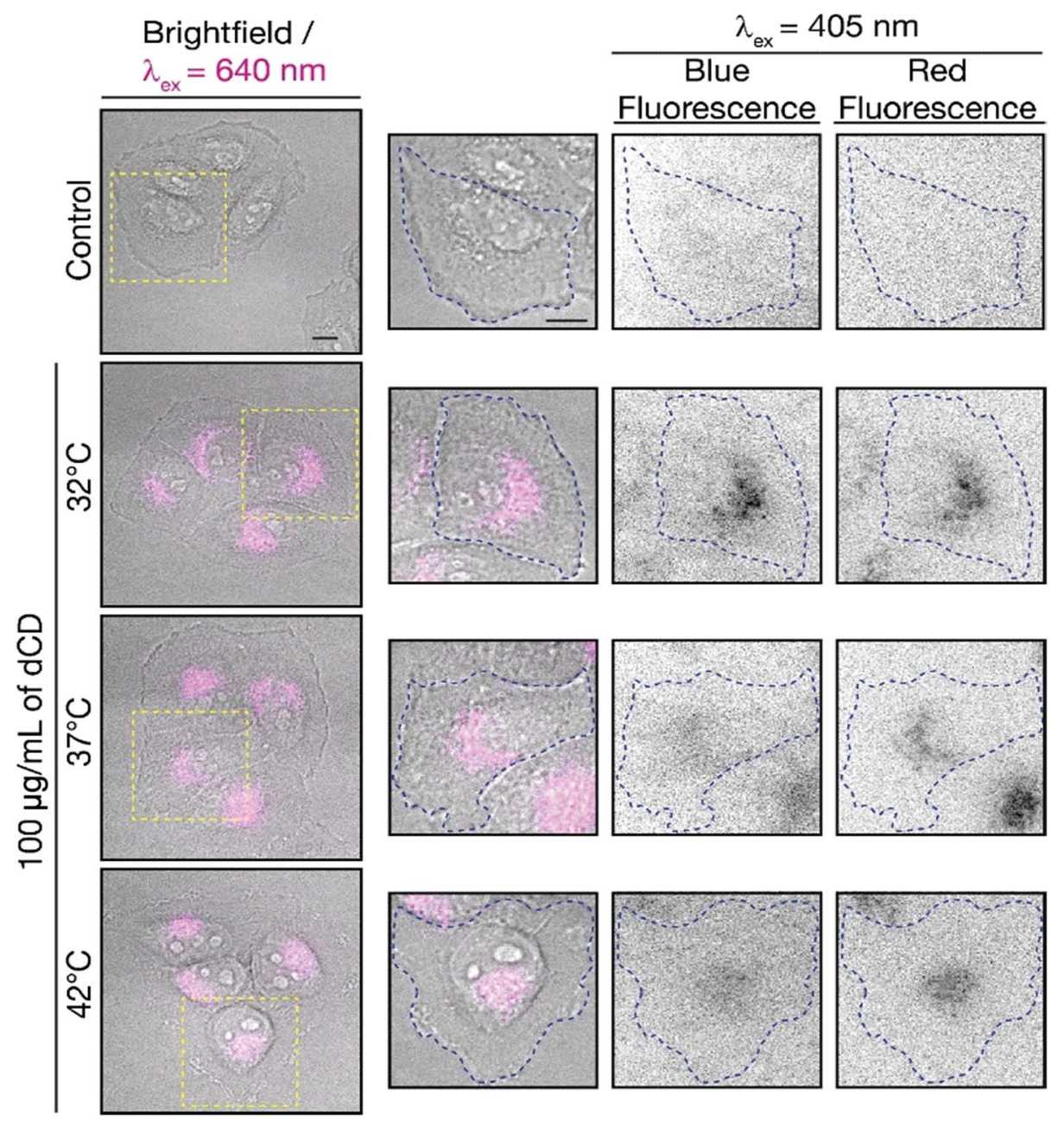

Fig. 5 Fluorescence microscopy images of dCD-treated HeLa cells. Fluorescence signals from the dCDs ( $\lambda_{\text {ex }}=640 \mathrm{~nm}$; left and $405 \mathrm{~nm}$; right) are shown for cells incubated at the different temperatures as indicated. The scale bars represent a length of $10 \mu \mathrm{m}$. The red-to-blue fluorescence ratios are 1.8 at $32{ }^{\circ} \mathrm{C}, 2.0$ at $37^{\circ} \mathrm{C}$ and 2.3 at $42{ }^{\circ} \mathrm{C}$. The control shows untreated HeLa cells at $42{ }^{\circ} \mathrm{C}$ with no fluorescence signal as expected.

HeLa cells, they were subjected to a heating/cooling cycle from $32 \rightarrow 42 \rightarrow 32{ }^{\circ} \mathrm{C}$. Rapid temperature changes were induced through the replacement of the cell media at the respective temperatures. Following analysis of an excess of 30 cells at each thermal event, the fluorescence properties remained unaffected with corresponding ratios of $1.5 \rightarrow 2.2 \rightarrow 1.7$. This highlights the robustness of the proposed dCD-nanothermometer and these findings further demonstrate the fluorescence reversibility as shown in Fig. S5.†

\section{Conclusion}

In this study, we describe the synthesis and physico-chemical properties of biocompatible dual-fluorescing carbon dots via the microwave reaction of formamide and glutathione. The water dispersible dCDs possess temperature-sensitive and reversible fluorescence. They show linear responses to the change in temperature from 5 to $60{ }^{\circ} \mathrm{C}$ in a cuvette model determined by intensity-based and ratiometric temperature sensing methods. These results translate into an in vitro cell model, where the ratiometric change in fluorescence was also observed in cells incubated at different temperatures. Unlike intensity-based measurements, temperature measurements using the ratiometric approach are not impacted by changes in dCD concentration, which could vary due to altered cellular uptake depending on cell density and/or temperature. With its facile synthesis, unique optical properties and biocompatibility, these dCDs are good candidates for in vivo nanothermometry in biological systems. Furthermore, they could be explored for bioimaging applications in the future.

\section{Experimental section}

\section{Chemicals and reagents}

Formamide ( $\geq 99.5 \%$ ) and reduced L-glutathione $(\geq 98.0 \%)$ were purchased from Thermo Scientific. Phosphate buffer solution (PBS, $1 \times$ ) and Dulbecco's modified Eagle medium (DMEM) were purchased from Wisent. HyClone ${ }^{\mathrm{TM}}$ Calf Serum and Tubulin 
Tracker Green ${ }^{\mathrm{TM}}$ were purchased from Thermo Scientific. The WST-8 Cell Proliferation Assay Kit was purchased from Cayman Chemical. All reagents were used without further modification or purification.

\section{Synthesis of dual-fluorescing CDs (dCDs)}

The dCDs were synthesized using a modified microwavemediated one-step reaction with glutathione and formamide as previously reported. ${ }^{36}$ Briefly, a $20 \mathrm{~mL}$ solution of $0.1 \mathrm{M}$ glutathione in formamide was prepared. The mixture was sonicated for $15 \mathrm{~min}$ until it changed from a cloudy to a clear homogeneous solution. The solution was then transferred to a $35 \mathrm{~mL}$ microwave reaction vial and heated to $180{ }^{\circ} \mathrm{C}$ for $5 \mathrm{~min}$. Once cooled, the dispersion was dialyzed using a cellulose ester membrane dialysis membrane (molecular weight cut-off $=3.5-$ $5.0 \mathrm{kDa}$ ) to remove unreacted materials and fluorophores. The sample was dialyzed for over five days with the water changed twice a day. Following this, the sample was passed through a $0.2 \mu \mathrm{m}$ nylon filter to remove any aggregates. Subsequently, the samples were washed twice with ethanol and twice with acetone $(1: 10$, sample : solvent volume ratio) to remove any remaining impurities. After each wash, the precipitate was collected by centrifugation at $10000 \times g$ for $10 \mathrm{~min}$. The resulting material was dried in the oven at $70{ }^{\circ} \mathrm{C}$ overnight and resuspended in water.

\section{Characterization techniques}

For Transmission Electron Microscopy (TEM), CDs were dispersed in water at a concentration of $5.0 \mathrm{mg} \mathrm{mL}^{-1}$. Grids were prepared by pipetting $2 \mu \mathrm{L}$ of the dCD dispersion onto a 200 mesh Formvar/carbon coated copper grid ( $3 \mathrm{~mm}$ in diameter) followed by evaporation of the solvent. The TEM images were collected using a Jeol JEM-2100F microscope operating at $100 \mathrm{kV}$. Images were processed and the carbon dot sizes were determined using Fiji imaging software. ${ }^{47}$ UV-visible absorption spectra were acquired from $200-800 \mathrm{~nm}$ on a Cary 5 Series UV-Vis-NIR Spectrophotometer (Agilent Technologies) using a $1 \mathrm{~cm}$ quartz cuvette. A $5.0 \mathrm{~nm}$ bandwidth and wavelength changeover at $450 \mathrm{~nm}$ were used for analysis. Data was processed using Cary Eclipse software. Quantum yield values were acquired on an FLS920 Fluorescence Spectrometer (Edinburgh Instruments) with an integrating sphere using a $1 \mathrm{~cm}$ quartz cuvette. The excitation and the emission slits were set to a width of $5 \mathrm{~nm}$. The excitation wavelength was set to $405 \mathrm{~nm}$ and the spectra from 300-800 $\mathrm{nm}$ were collected. Scans were done in triplicates with a dwell time of $0.2 \mathrm{~s}$. Data was processed using F900 Software. Fluorescence spectra were acquired using a Cary Eclipse fluorescence spectrophotometer (Agilent Technologies). Spectra were acquired in a $1 \mathrm{~cm}$ quartz cuvette at $\lambda_{\text {ex }}=360-660 \mathrm{~nm}$ (5 nm intervals). The excitation and emission slits were set to a width of $5 \mathrm{~nm}$ with a PMT voltage at $600 \mathrm{~V}$. All data were processed using Cary Eclipse software. To obtain fluorescence spectra at various temperatures, the Cary Single Cell Peltier Accessory (Agilent Technologies) was used to adjust the temperature from $5{ }^{\circ} \mathrm{C}$ to $60{ }^{\circ} \mathrm{C}$. Fourier-Transform Infrared Spectroscopy (FT-IR) spectra were collected using a Thermo Scientific Nicolet iS5 equipped with an iD5 ATR accessory. Spectra were collected using 30 scans with a resolution of $0.4 \mathrm{~cm}^{-1}$, a gain of 1 , an optical velocity 0.4747 and an aperture setting of 100 . Data was processed using Omnic 9 software. X-ray Photoelectron Spectroscopy (XPS) spectra of the dCDs were acquired using a Thermo Scientific K-Alpha X-ray Photoelectron Spectrometer. Each analysis was carried out in triplicate with 10 runs for each scan. The averages were plotted for both the survey and highresolution scans. The stability of the dCDs $\left(50 \mu \mathrm{g} \mathrm{mL} \mathrm{m}^{-1}\right)$ was evaluated by measuring the fluorescence intensities from the same stock solution of dCDs once a month over a span of 6 months. Fluorescence intensity $\left(\lambda_{\mathrm{ex}}=640 \mathrm{~nm}\right)$ centered around $680 \mathrm{~nm}$ was collected and used for the assessment.

\section{Cell culture and WST-8 assays}

HeLa cells were cultured in DMEM containing 10\% calf serum and maintained in a humidified incubator set to $37^{\circ} \mathrm{C}$ with $5 \%$ $\mathrm{CO}_{2}$. To assess cell viability, HeLa cells were plated in 96-well plates at $20 \%$ confluency and cultured overnight. The cytotoxicity of the dCDs was assessed via WST-8 cell proliferation assays (Cayman Chemical). The cells were treated with dCDs at varying concentrations ( 0 to $1000 \mathrm{~g} \mathrm{~mL}^{-1}$ ) and incubated for 48 hours. Subsequently, the WST- 8 reagent was added for 4 hours. The optical density (OD) values were measured using the TECAN 200 PRO plate reader at a wavelength of $490 \mathrm{~nm}$. Cell viability was measured as a ratio of the signal of treated cells $v s$. control:

$$
\% \text { cell viability }=\frac{\mathrm{OD}(490 \mathrm{~nm})_{\text {sample }}}{\mathrm{OD}(490 \mathrm{~nm})_{\text {blank }}} \times 100 \%
$$

All measurements were repeated in triplicate and the means were plotted with standard deviation.

\section{Cellular imaging}

HeLa cells were imaged after treatment with the dCDs. Cells were plated in Nunc ${ }^{\mathrm{TM}}$ Lab-Tek II 4-well chambered microslides (Thermo Scientific ${ }^{\mathrm{TM}}$ ) and left overnight to adhere. The dCDs were added to cells in media at a final concentration of $100 \mu \mathrm{g}$ $\mathrm{mL}^{-1}$ for 24 hours. To determine the localization of dCDs, HeLa cells were treated with TubulinTracker Green Reagent (Molecular Probes) at a final concentration of $250 \mathrm{nM}$ as per manufacturer's instructions to stain microtubules and Hoechst 33342 dye (Thermo Scientific ${ }^{\mathrm{TM}}$ ) at a final concentration of $75 \mathrm{nM}$ to stain the DNA. The cells were then placed in the incubator at $37^{\circ} \mathrm{C}$ with $5 \% \mathrm{CO}_{2}$ for 30 minutes after which the media was replaced. Imaging was performed using the Nikon-TIE inverted epifluorescence microscope with Lambda XL LED light sources using the $60 \times / 1.4$ oil objective, a Piezo Z stage (ASI), a Photometrics Evolve 512 EMCCD camera and Elements 4.0 acquisition software (Nikon). Images were captured as $0.5 \mu \mathrm{m} Z$-stacks and converted into maximum intensity $Z$-stack projections using FIJI (NIH).

\section{Intracellular nanothermometry}

To measure the nanothermometric properties of the dCDs, HeLa cells were prepared as described above. In each Lab-Tek II 
chamber, three wells containing Hela cells were treated with $100 \mu \mathrm{g} \mathrm{mL}{ }^{-1}$ of dCDs and placed at $32{ }^{\circ} \mathrm{C}, 37^{\circ} \mathrm{C}$ or $42{ }^{\circ} \mathrm{C}$ for 2 hours prior to imaging $\left(n_{\mathrm{i}}=30\right.$ cells). The controls were subjected to the same conditions, except without addition of the dCDs. After 2 hours, the wells were placed into an INU-TiZ-F1 chamber (MadCityLabs) with $5 \% \quad \mathrm{CO}_{2}$ and heated at their respective experimental temperature for imaging, which was mounted on the stage on the inverted microscope. Imaging was carried out using the same microscope described above. Excitation at 405 or $640 \mathrm{~nm}$ was used to capture emission at $460 \mathrm{~nm}$ using a 400-600 $\mathrm{nm}$ bandpass (Chroma CT500/200bp) filter, or at $680 \mathrm{~nm}$ using a $610 \mathrm{~nm}$ longpass (Chroma AT610lp) filter, respectively. Images were acquired as $0.5 \mu \mathrm{m} Z$-stacks using Elements 4.0 software (Nikon) and converted into maximum intensity $Z$-stack projections using FIJI (NIH). Background intensities were subtracted using the control. In vitro intracellular temperature was monitored following a thermal cycling regime to assess the reversibility of the fluorescence properties. Initially, the dCD-treated cells were incubated at $32{ }^{\circ} \mathrm{C}$. Then, the temperature was rapidly cycled by replacing the media with media already equilibrated at $42{ }^{\circ} \mathrm{C}$. Finally, the temperature was cycled down to $32{ }^{\circ} \mathrm{C}$ once again by replacing the media. The red-to-blue emission ratios were calculated at each temperature.

\section{Conflicts of interest}

There are no conflicts to declare.

\section{Acknowledgements}

The authors would like to acknowledge funding sources for financial support for this research. R. N. is grateful to NSERC for funding through the Discovery Grant and Discovery Accelerator Supplement. R. N. is also grateful to Concordia University for funding through Start-up and Strategic funds. AP is funded by NSERC Discovery and Accelerator Supplement grants, as well as FQRNT. TEM work was performed in Centre (CM)2 at Universite de Montreal with the assistance of Dr Jean-Philippe Masse. AFM work was performed at the NanoQAM facilities at the Universite du Québec à Montréal with the assistance of Mr Gwenaël Chamoulaud. XPS studies were performed in McGill University (MIAM Facilities in the Department of Mining and Materials Engineering) with the assistance of Dr Lihong Shang. Microscopy images were taken with the assistance of Dr Chris Law at the Centre for Microscopy and Cellular Imaging at Concordia University (CMCI).

\section{References}

1 M. Suzuki, V. Tseeb, K. Oyama and S. Ishiwata, Biophys. J., 2007, 92, L46-L48.

2 O. Zohar, M. Ikeda, H. Shinagawa, H. Inoue, H. Nakamura, D. Elbaum, D. L. Alkon and T. Yoshioka, Biophys. J., 1998, 74, 82-89.

3 d. R. Blanca, X. Erving, R. Ueslen and J. Daniel, Adv. Opt. Mater., 2017, 5, 1600508.
4 D. Jaque and F. Vetrone, Nanoscale, 2012, 4, 4301-4326.

5 D. Ruiz, B. del Rosal, M. Acebrón, C. Palencia, C. Sun,

J. Cabanillas-González, M. López-Haro, A. B. Hungría,

D. Jaque and B. H. Juarez, Adv. Funct. Mater., 2017, 27, 1604629.

6 X. Chen, S. Yu, L. Yang, J. Wang and C. Jiang, Nanoscale, 2016, 8, 13669-13677.

7 Y. Wang, C. Zhang, X. Chen, B. Yang, L. Yang, C. Jiang and Z. Zhang, Nanoscale, 2016, 8, 5977-5984.

8 J. Zhang, L. He, P. Chen, C. Tian, J. Wang, B. Liu, C. Jiang and Z. Zhang, Nanoscale, 2017, 9, 1599-1606.

9 H. Liu, Y. Fan, J. Wang, Z. Song, H. Shi, R. Han, Y. Sha and Y. Jiang, Sci. Rep., 2015, 5, 14879.

10 F. Vetrone, R. Naccache, A. Zamarrón, A. Juarranz de la Fuente, F. Sanz-Rodríguez, L. Martinez Maestro, E. Martín Rodriguez, D. Jaque, J. García Solé and J. A. Capobianco, ACS Nano, 2010, 4, 3254-3258.

11 I. E. Kolesnikov, E. V. Golyeva, M. A. Kurochkin, E. Lähderanta and M. D. Mikhailov, Sens. Actuators, B, 2016, 235, 287-293.

12 C. D. S. Brites, P. P. Lima, N. J. O. Silva, A. Millan, V. S. Amaral, F. Palacio and L. D. Carlos, Nanoscale, 2012, 4, 4799-4829.

13 C. D. S. Brites, P. P. Lima, N. J. O. Silva, A. Millan, V. S. Amaral, F. Palacio and L. D. Carlos, New J. Chem., 2011, 35, 1177-1183.

14 S. Balabhadra, M. L. Debasu, C. D. S. Brites, R. A. S. Ferreira and L. D. Carlos, J. Phys. Chem. C, 2017, 121, 13962-13968.

15 K. Okabe, N. Inada, C. Gota, Y. Harada, T. Funatsu and S. Uchiyama, Nat. Commun., 2012, 3, 705.

16 S. Kalytchuk, K. Poláková, Y. Wang, J. P. Froning, K. Cepe, A. L. Rogach and R. Zbořil, ACS Nano, 2017, 11, 1432-1442.

17 A. Carattino, M. Caldarola and M. Orrit, Nano Lett., 2018, 18, 874-880.

18 A. Cadiau, C. D. S. Brites, P. M. F. J. Costa, R. A. S. Ferreira, J. Rocha and L. D. Carlos, ACS Nano, 2013, 7, 7213-7218.

19 E. Y. K. Ng, Int. J. Therm. Sci., 2009, 48, 849-859.

20 N. S. Abadeer and C. J. Murphy, J. Phys. Chem. C, 2016, 120, 4691-4716.

21 B. Thiesen and A. Jordan, Int. J. Hyperthermia, 2008, 24, 467474.

22 F. Jonathan and C. Yu-Chie, Curr. Pharm. Des., 2013, 19, 6622-6634.

23 V. Nguyen, L. Yan, H. Xu and M. Yue, Appl. Surf. Sci., 2018, 427, 1118-1123.

24 Y. Yang, W. Kong, H. Li, J. Liu, M. Yang, H. Huang, Y. Liu, Z. Wang, Z. Wang, T.-K. Sham, J. Zhong, C. Wang, Z. Liu, S.-T. Lee and Z. Kang, ACS Appl. Mater. Interfaces, 2015, 7, 27324-27330.

25 S. Y. Lim, W. Shen and Z. Gao, Chem. Soc. Rev., 2015, 44, 362381.

26 M. L. Bhaisare, A. Talib, M. S. Khan, S. Pandey and H.-F. Wu, Microchim. Acta, 2015, 182, 2173-2181.

27 Y.-P. Sun, B. Zhou, Y. Lin, W. Wang, K. A. S. Fernando, P. Pathak, M. J. Meziani, B. A. Harruff, X. Wang, H. Wang, P. G. Luo, H. Yang, M. E. Kose, B. Chen, L. M. Veca and S.-Y. Xie, J. Am. Chem. Soc., 2006, 128, 7756-7757. 
28 S. Zhu, Y. Song, X. Zhao, J. Shao, J. Zhang and B. Yang, Nano Res., 2015, 8, 355-381.

29 Y. Wang and A. Hu, J. Mater. Chem. C, 2014, 2, 6921-6939.

30 H. Ding, J.-S. Wei, N. Zhong, Q.-Y. Gao and H.-M. Xiong, Langmuir, 2017, 33, 12635-12642.

31 W. Lu, X. Gong, Z. Yang, Y. Zhang, Q. Hu, S. Shuang, C. Dong and M. M. F. Choi, RSC Adv., 2015, 5, 16972-16979.

32 C. Yang, S. Zhu, Z. Li, Z. Li, C. Chen, L. Sun, W. Tang, R. Liu, Y. Sun and M. Yu, Chem. Commun., 2016, 52, 11912-11914.

33 S. Sun, L. Zhang, K. Jiang, A. Wu and H. Lin, Chem. Mater., 2016, 28, 8659-8668.

34 L. Pan, S. Sun, A. Zhang, K. Jiang, L. Zhang, C. Dong, Q. Huang, A. Wu and H. Lin, Adv. Mater., 2015, 27, 7782-7787.

35 X. Liu, X. Tang, Y. Hou, Q. Wu and G. Zhang, RSC Adv., 2015, 5, 81713-81722.

36 K. Jiang, J. Wu, Q. Wu, X. Wang, C. Wang and Y. Li, Part. Part. Syst. Charact., 2017, 34, 1600197.

37 C. Wang, K. Jiang, Q. Wu, J. Wu and C. Zhang, Chem.-Eur. J., 2016, 22, 14475-14479.

38 C. Wang, H. Lin, Z. Xu, Y. Huang, M. G. Humphrey and C. Zhang, ACS Appl. Mater. Interfaces, 2016, 8, 6621-6628.

39 W. Zhou, J. Zhuang, W. Li, C. Hu, B. Lei and Y. Liu, J. Mater. Chem. C, 2017, 5, 8014-8021.

40 R. Mohan, J. Drbohlavova and J. Hubalek, Chem. Phys. Lett., 2018, 692, 196-201.

41 D. Chen, H. Gao, X. Chen, G. Fang, S. Yuan and Y. Yuan, ACS Photonics, 2017, 4, 2352-2358.

42 L. Pan, S. Sun, L. Zhang, K. Jiang and H. Lin, Nanoscale, 2016, 8, 17350-17356.
43 D. Qu, M. Zheng, P. Du, Y. Zhou, L. Zhang, D. Li, H. Tan, Z. Zhao, Z. Xie and Z. Sun, Nanoscale, 2013, 5, 12272-12277.

44 L. Tang, R. Ji, X. Cao, J. Lin, H. Jiang, X. Li, K. S. Teng, C. M. Luk, S. Zeng, J. Hao and S. P. Lau, ACS Nano, 2012, 6, 5102-5110.

45 K. König, J. Microsc., 2000, 200, 83-104.

46 K. König and H. Schneckenburger, J. Fluoresc., 1994, 4, 17-40.

47 H. Y. Ko, Y. W. Chang, G. Paramasivam, M. S. Jeong, S. Cho and S. Kim, Chem. Commun., 2013, 49, 10290-10292.

48 T. Huiquan, Y. Kai, M. Zhen, W. Jianmei, Z. Youjiu, K. Zhenhui and L. Zhuang, Small, 2012, 8, 281-290.

49 S. Lu, L. Sui, J. Liu, S. Zhu, A. Chen, M. Jin and B. Yang, Adv. Mater., 2017, 29, 1603443.

50 D. Jaque, B. d. Rosal, E. M. Rodríguez, L. M. Maestro, P. Haro-González and J. G. Solé, Nanomedicine, 2014, 9, 1047-1062.

51 E. J. McLaurin, V. A. Vlaskin and D. R. Gamelin, J. Am. Chem. Soc., 2011, 133, 14978-14980.

52 A. E. Albers, E. M. Chan, P. M. McBride, C. M. Ajo-Franklin, B. E. Cohen and B. A. Helms, J. Am. Chem. Soc., 2012, 134, 9565-9568.

53 C. Yuanjing, S. Ruijing, Y. Jiancan, L. Min, W. Ziqi, W. Chuande, Y. Yu, W. Zhiyu, C. Banglin and Q. Guodong, Adv. Mater., 2015, 27, 1420-1425.

54 J. Llopis, J. M. McCaffery, A. Miyawaki, M. G. Farquhar and R. Y. Tsien, Proc. Natl. Acad. Sci. U. S. A., 1998, 95, 6803-6808. 